\title{
Dynamic formation of quasicondensate and spontaneous vortices in a strongly interacting Fermi gas
}

\author{
Xiang-Pei Liu, ${ }^{1,2,3,{ }^{*}}$ Xing-Can Yao $\odot, 1,2,3,{ }^{*}$ Youjin Deng, $, 1,2,3,6,{ }^{*}$ Yu-Xuan Wang, ${ }^{1,2,3}$ Xiao-Qiong Wang, ${ }^{1,2,3}$ \\ Xiaopeng Li $\odot,{ }^{4,3,5}$ Qijin Chen, ${ }^{1,2,3}$ Yu-Ao Chen $\odot,{ }^{1,2,3}$ and Jian-Wei Pan ${ }^{1,2,3}$ \\ ${ }^{1}$ Hefei National Laboratory for Physical Sciences at the Microscale and Department of Modern Physics, University of Science and \\ Technology of China, Hefei 230026, China \\ ${ }^{2}$ Shanghai Branch, CAS Center for Excellence in Quantum Information and Quantum Physics, University of Science and Technology of \\ China, Shanghai 201315, China \\ ${ }^{3}$ Shanghai Research Center for Quantum Sciences, Shanghai 201315, China \\ ${ }^{4}$ State Key Laboratory of Surface Physics, Institute of Nanoelectronics and Quantum Computing, and Department of Physics, Fudan \\ University, Shanghai 200433, China \\ ${ }^{5}$ Shanghai Qi Zhi Institute, AI Tower, Xuhui District, Shanghai 200232, China \\ ${ }^{6}$ MinJiang Collaborative Center for Theoretical Physics, College of Physics and Electronic Information Engineering, \\ Minjiang University, Fuzhou 350108, China
}

(Received 28 July 2021; accepted 2 November 2021; published 15 November 2021)

\begin{abstract}
We report an experimental study of quench dynamics across the superfluid transition temperature $T_{\mathrm{c}}$ in a strongly interacting Fermi gas by ramping down the trapping potential. The nonzero quasicondensate number $N_{0}$ at temperatures significantly above $T_{\mathrm{c}}$ in the unitary and the BEC regimes is consistent with the pseudogap physics via preformed pairs. Below $T_{\mathrm{c}}$, a rapid growth of $N_{0}$ is accompanied by the spontaneous generation of tens of vortices. We observe a power-law scaling of the vortex density versus the quasicondensate formation time, consistent with the Kibble-Zurek theory. Our work provides an example of studying emerged many-body physics by quench dynamics and paves the way for studying the quantum turbulence in a strongly interacting Fermi gas.
\end{abstract}

DOI: 10.1103/PhysRevResearch.3.043115

\section{INTRODUCTION}

In pursuit of correlated quantum physics in strongly interacting Fermi gases, great efforts have been devoted to studying equilibrium phases and transitions [1-6]. This has shed light on the understanding of high- $T_{\mathrm{c}}$ superconductivity [7-9] and the modeling of equation of states of dense neutron stars [10]. Of equal importance would be to probe the nonequilibrium dynamics during a temperature quench across the superfluid transition temperature $T_{c}$, where the superfluid growth is closely connected to the generation of spontaneous vortices.

For bosonic systems, the quench dynamics has been intensively studied [11-17] and can be well described by the Kibble-Zurek (KZ) theory $[18,19]$. Very recently, the observation of KZ scaling was also reported in Fermi gases [20]. However, it is expected that the quench dynamics of strongly interacting Fermi systems should possess much richer physics due to the complexity of fermionic superfluid formation. Fermionic atoms have to pair into bosonic degrees of free-

\footnotetext{
${ }^{*}$ These authors contributed equally to this work.

Published by the American Physical Society under the terms of the Creative Commons Attribution 4.0 International license. Further distribution of this work must maintain attribution to the author $(s)$ and the published article's title, journal citation, and DOI.
}

dom, Cooper pairs or bound molecules, for the formation of a superfluid. In addition to the transition temperature $T_{\mathrm{c}}$, there exists another characteristic temperature $T^{*}$, characterizing the onset of pair formation. In the weak-coupling BCS limit, pair formation and pair condensation occur essentially at the same temperature, leading to a rapid growth of superfluid fraction as the temperature $T$ is lowered across $T_{\mathrm{c}}$. However, as the pairing strength increases, these two temperatures become distinct, and pairs can preform far above $T_{\mathrm{c}}$. This leads to a pseudogap in the fermionic excitation spectrum. At the same time, isolated superfluid islands having random relative phases may also appear above $T_{\mathrm{c}}$. As the temperature decreases, they may merge to generate vortices spontaneously. Finally, superfluidity with global phase coherence is gradually established with the annihilation of these vortices and antivortices. Therefore, the quench dynamics offers a great opportunity for understanding the interplay among the formation of bosonic pairs, superfluid phase coherence, and spontaneous vortices.

Here, we report an experimental study of the real-time dynamics of superfluid growth and spontaneous vortex formation in a strongly interacting Fermi gas of ${ }^{6} \mathrm{Li}$ atoms. We rapidly ramp down the potential of the oblate optical trap so that the system is effectively thermally quenched across the superfluid transition. For a given ramping time, the quasicondensate number $N_{0}$ (consisting of bosonic pairs in the vicinity of zero momentum) is recorded in real time, while the spontaneously generated vortex density $\rho_{v}$ is measured 


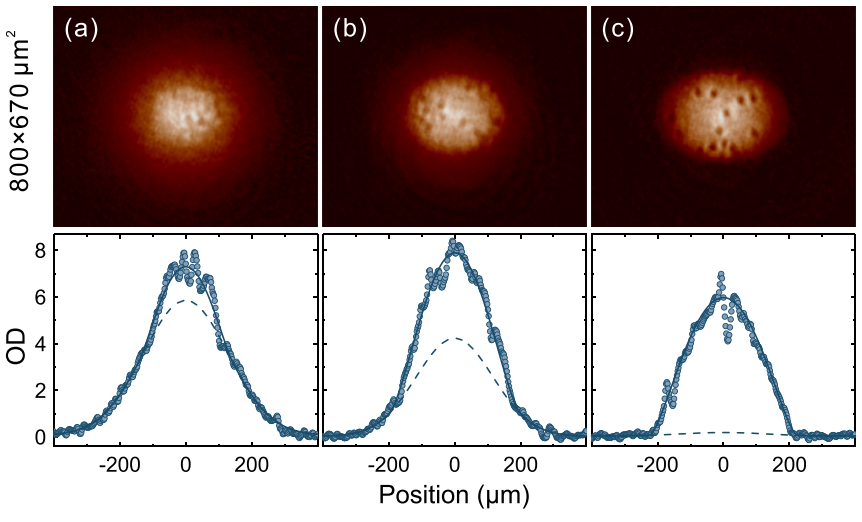

FIG. 1. Illustration of the real-time formation dynamics of quasicondensate and spontaneous vortices, at unitarity $(832 \mathrm{G})$ with $t_{\text {ramp }}=600 \mathrm{~ms}$. The top row shows the absorption images of the cloud after 10-ms time of flight (TOF) at $t=520,580$, and $700 \mathrm{~ms}$, respectively, from left to right. Here, $t$ is defined as the evolution time of the system, i.e., $t=0$ marks the start of the quench. Plots in the bottom row are central line cuts of the column density distribution. The solid lines are the fits with a Gaussian plus Thomas-Fermi distribution, and the dashed lines indicate the Gaussian part alone. The fitting yields the quasicondensate fraction $N_{0}(t) / N_{0 \text {, sat }} \approx 0.1$, 0.5 , and 1 (from left to right), corresponding to the initial increase, rapid growth, and saturation stages of the quasicondensate number, respectively.

upon $N_{0}$ reaching saturation. The observed growth dynamics of $N_{0}$ agree with calculations based on the pairing fluctuation theory [21,22], by assuming that the system temperature $T$ decreases linearly with the evolution time $t$ during the ramp. The evolution of the growth dynamics of $N_{0}$ is consistent with the pseudogap physics via preformed pairs throughout the BCS-BEC crossover. At unitarity, for normal quenches with a ramping time $t_{\text {ramp }} \geqslant 600 \mathrm{~ms}$, the quasicondensate formation time $t_{\mathrm{f}}$ linearly increases with $t_{\mathrm{ramp}}$ and the growth dynamics of $N_{0}$ nicely collapse onto a single universal curve. In contrast, for fast quenches with $t_{\text {ramp }} \leqslant 400 \mathrm{~ms}$, $t_{\mathrm{f}}$ drops significantly as $t_{\text {ramp }}$ increases, and the growth curves of $N_{0}$ exhibit a significant deviation from the collapse, both of which hint at the breakdown of the quasiequilibrium condition. Furthermore, by using $t_{\mathrm{f}}$ as the quench time, which is less sensitive to the pseudogap physics, a power-law scaling of $\rho_{v}$ vs $t_{\mathrm{f}}$ is observed for normal quenches, and the extracted critical exponent agrees quantitatively with that predicted by the $\mathrm{KZ}$ theory.

\section{EXPERIMENTAL METHOD}

The main experimental setup and method for preparing the ${ }^{6} \mathrm{Li}$ superfluid have been described in our previous works [23]. We start by preparing a spin-balanced mixture of $1 \times 10^{7}$ atoms at $832.18 \mathrm{G}$ in an elliptical optical dipole trap $\left[1 / e^{2}\right.$ radius $200 \mu \mathrm{m}$ and $48 \mu \mathrm{m}$ (in the gravity direction)]. Further evaporative cooling is performed by ramping down the trap depth and holding for $3 \mathrm{~s}$, yielding a superfluid of $3.9(1) \times$ $10^{6}$ atoms at about $0.3 T_{\mathrm{c}}$. With a short ramping time, i.e., $t_{\mathrm{ramp}}$ varies from 200 to $1500 \mathrm{~ms}$, a temperature quench across the

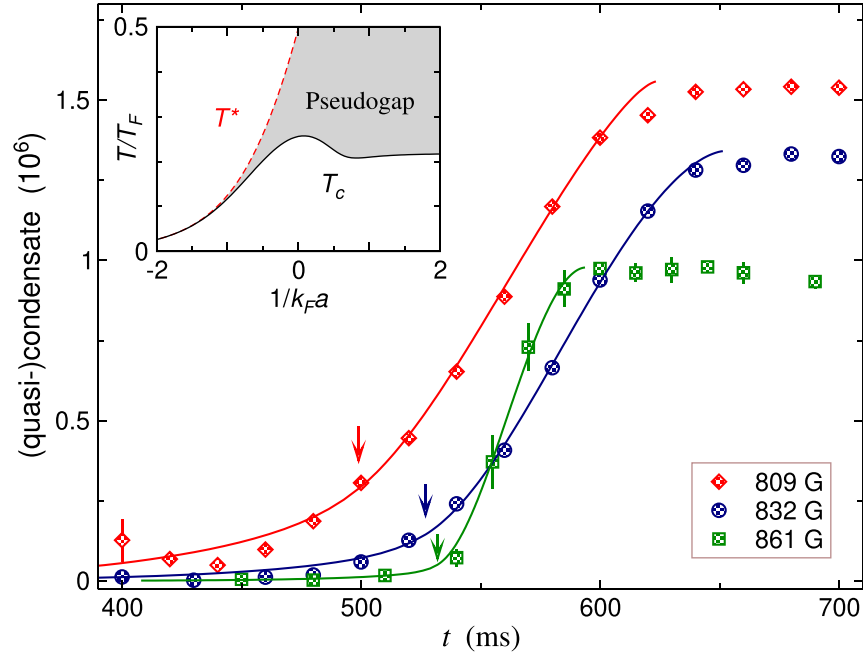

FIG. 2. Real-time dynamics of quasicondensate number $N_{0}$ with $t_{\text {ramp }}=600 \mathrm{~ms}$, at the magnetic field of $809 \mathrm{G}$ (red diamonds, BEC side), $832 \mathrm{G}$ (blue circles, unitarity), and $861 \mathrm{G}$ (green squares, BCS side). Each data point is an average of about ten individual measurements with standard statistical error. The curves represent the theoretical results calculated based on the pairing fluctuation theory, which have been rescaled and horizontally shifted to fit the experimental data. The arrows indicate locations of superfluid transition from theory. The inset shows the phase diagram of a 3D homogeneous Fermi gas in the BCS-BEC crossover as a function of $1 / k_{\mathrm{F}} a$, which manifests a pseudogap region between $T_{\mathrm{c}}$ and $T^{*}$. Here, $k_{\mathrm{F}}$ and $a$ denote the Fermi momentum and the $s$-wave scattering length, respectively.

superfluid transition can be achieved, during which plenty of vortices are spontaneously generated $[12,13,15]$.

To probe the quasicondensate and vortices, the optical trap is suddenly switched off and the magnetic field is rapidly ramped to $720 \mathrm{G}$. After expansion for a total time of $10 \mathrm{~ms}$, strong saturation absorption imaging along the gravity direction is performed. The quasicondensate number $N_{0}$ is then obtained by fitting the density profile of the cloud with a Gaussian plus Thomas-Fermi distribution. The dynamic formation of vortices is clearly visible, as shown in Fig. 1 . When $N_{0}$ is small, the vortex cores are blurred with very low contrast and are distributed in a small spatial region. As $N_{0}$ increases, the vortices become more visible and spread over the entire cloud. This gives a direct and vivid illustration of the evolution of superfluid coherence and the formation of spontaneous vortices. We mention that owing to the oblate trap geometry, the cloud expands rapidly in the gravity direction, resulting in a reduced imaging resolution. Nevertheless, upon saturation of $N_{0}$, a high contrast of vortex cores is still achieved [see Fig. 1(c)], suggesting a straight alignment of the vortex lines.

\section{QUASICONDENSATE GROWTH IN THE BCS-BEC CROSSOVER}

We first investigate the growth dynamics of the quasicondensate in the BCS-BEC crossover for $t_{\mathrm{ramp}}=600 \mathrm{~ms}$. Figure 2 shows the quasicondensate number $N_{0}$ as a function of $t$ for three typical magnetic fields of 809,832 , and $861 \mathrm{G}$. 

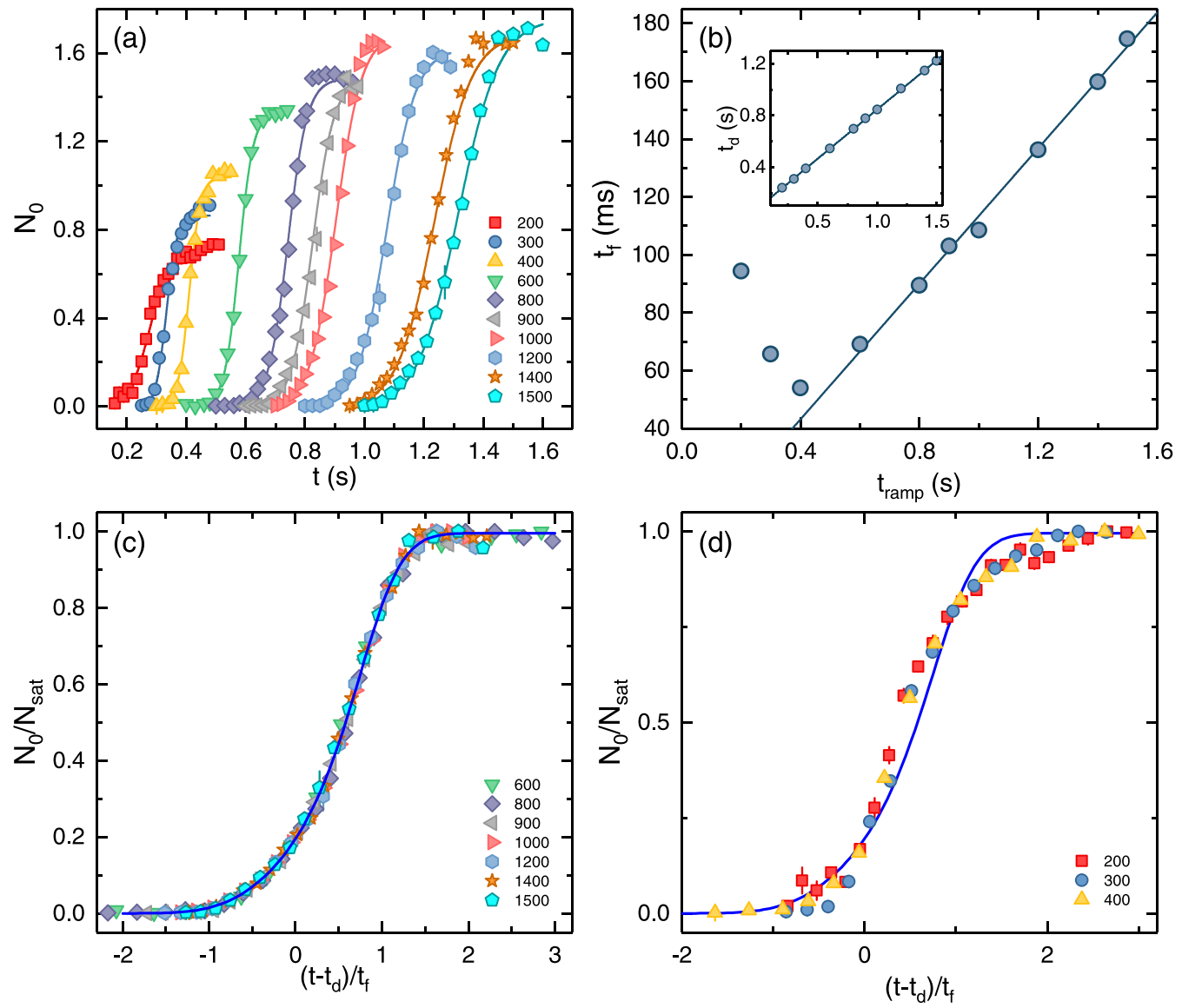

FIG. 3. Real-time dynamics of the quasicondensate $N_{0}$ at unitarity. Each data point represents an average of about ten individual measurements with the standard error bar. (a) Growth curves with $t_{\text {ramp }}$ ranging from 200 to 1500 ms, where the solid curves are guides to the eye. (b) Formation time $t_{\mathrm{f}}$ and delay time $t_{\mathrm{d}}$ (inset) vs $t_{\text {ramp. }}$. Solid lines are linear fittings. (c) $N_{0} / N_{0 \text {, sat }}$ vs $\left(t-t_{\mathrm{d}}\right) / t_{\mathrm{f}}$, for normal quenches with $t_{\text {ramp }} \geqslant 600 \mathrm{~ms}$. The data are fitted with a smoothing spline (solid line). (d) $N_{0} / N_{0 \text {,sat }}$ data for fast quenches with $t_{\text {ramp }} \leqslant 400 \mathrm{~ms}$, compared with the solid curve for normal quenches.

Here, $t$ is the evolution time of the system, starting at the beginning of the quench. All three $N_{0}$ curves seem to have a similar shape, with an initial slow increase, followed by a rapid condensate formation, and finally a nearly flat saturation. A closer look at the growth of $N_{0}$ reveals the qualitative difference as the magnetic field increases. In the initial slow increase phase, $N_{0 \text {,ini }}$ is clearly nonzero at $809 \mathrm{G}$ (BEC) and $832 \mathrm{G}$ (unitarity), while it remains nearly zero for $861 \mathrm{G}$ (BCS). During the rapid growth stage, the formation rate of the quasicondensate monotonically increases from the BEC to the BCS regimes [24].

To better understand the dependence of the $N_{0}$ growth on the interaction strength (magnetic field), we numerically calculate the equilibrium quasicondensate number $N_{0}^{\text {th }}$ based on the pairing fluctuation theory [25]. The pair dispersion $\Omega_{\mathbf{q}} \approx$ $\hbar^{2} q^{2} / 2 M-\mu_{\text {pair }}$, or equivalently the effective pair mass $M$ and the chemical potential $\mu_{\text {pair }}$, can be extracted from the pair propagator or the particle-particle scattering $\mathbb{T}$ matrix. Given the temperature and interaction strength, we are able to calculate the fermionic chemical potential $\mu$, the pairing gap $\Delta$, and the superfluid order parameter $\Delta_{s c}$ in the trap using the local density approximation. Note that the measured quasicondensate number contains bosonic pairs with both zero and small finite momenta. Thus, we choose a small energy cutoff $\Omega_{\mathrm{c}}$, and obtain the density profile of the quasicondensate $n_{0}(r)$ by summing over all the pairs with energy $\hbar^{2} q^{2} / 2 M<\Omega_{\mathrm{c}}$, i.e., $n_{0}(r)=\int_{q<\Lambda} \frac{d^{3} q}{(2 \pi)^{3}} b\left(\Omega_{\mathbf{q}}(r)\right)$, where $b(x)=1 /\left(e^{x / k_{B} T}-1\right)$ is the Bose distribution function and the cutoff $\Lambda=\sqrt{2 M \Omega_{\mathrm{c}}}$. Here, the energy cutoff is simply taken as $\Omega_{\mathrm{c}}=k_{B} T / 2$, in accordance with the experimental measurements [26]. Finally, we obtain the quasicondensate number $N_{0}=\int d^{3} r n_{0}(r)$ as a function of $T$.

To compare with the experimental growth dynamics of $N_{0}$, we assume a simple linear relation between evolution time $t$ and temperature $T$ before $N_{0}$ saturates at very low $T$, especially during the condensate formation stage. The theory curves are scaled in a way to match the saturation value $N_{0 \text {,sat }}$ at low $T$ and the slope at half saturation of their experimental counterpart. The arrows in Fig. 2 indicate the superfluid transition from theory, which correspond to a "critical time" $t_{\mathrm{c}}$, when the temperature crosses $T_{\mathrm{c}}$ in the evolution of the quench dynamics. It is known that, above $T_{\mathrm{c}}$, a pseudogap in the fermionic excitation spectrum can emerge and bosonic pairs of fermionic atoms can already preform. The pair-formation temperature $T^{*}$ depends on the atom-atom interaction. For illustrative purposes, the phase diagram for a three-dimensional 
(3D) homogeneous Fermi gas is shown in the inset of Fig. 2, where a pseudogap region is present between $T_{\mathrm{c}}$ and $T^{*}$. In general, $T^{*}$ is above $T_{\mathrm{c}}$, except in the BCS limit, where the two temperatures merge. In the unitary and the BEC regimes, a small but nonzero quasicondensate already forms before the critical time $t_{\mathrm{c}}$ or above $T_{\mathrm{c}}$. Since the correlation length $\xi$ is small above $T_{\mathrm{c}}$, the superfluid coherence is yet to be established over large distances, and hence the growth of $N_{0}$ is slow. As $T$ is lowered across $T_{\mathrm{c}}$ (or equivalently $t>t_{\mathrm{c}}$ ), $\xi$ can be as large as the linear size of the system, so that $N_{0}$ enters a rapid-growth period until its saturation. In contrast, in the BCS regime, where the pseudogap is absent, the pair formation and pair condensation roughly occur at the same temperature. As a result, $N_{0}$ remains nearly zero during the initial slow increase stage before entering an abrupt rapid growth immediately after $t_{\mathrm{c}}$, as seen in the experimental data at $861 \mathrm{G}$. Therefore, our experiment can be naturally explained by the pseudogap physics described in the theory.

Next, we study the dependence of the quench dynamics

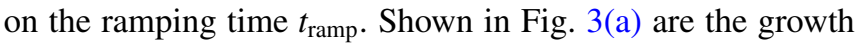
curves of $N_{0}$ at unitarity for $t_{\text {ramp }}$ ranging from 200 to $1500 \mathrm{~ms}$. As $t_{\text {ramp }}$ becomes longer, the saturated quasicondensate number $N_{0 \text {,sat }}$ also increases because of less atom loss during the evaporative cooling. It is seen that for quenches with $t_{\text {ramp }} \geqslant 600 \mathrm{~ms}, N_{0}$ roughly reaches its saturation at the end of quench. In contrast, for quenches with $t_{\text {ramp }} \leqslant 400 \mathrm{~ms}$, the rapid formation of $N_{0}$ has barely started by $t=t_{\text {ramp }}$, and the much suppressed $N_{0 \text {, sat }}$ is not reached until a much later time. To better describe the quench dynamics, we introduce two timescales, delay time $t_{\mathrm{d}}$ and formation time $t_{\mathrm{f}}$, corresponding to the starting time and the duration of the rapid formation of $N_{0}$, respectively. In practice, they are determined via $N_{0}\left(t_{\mathrm{d}}\right) / N_{0 \text {, sat }}=0.2$ and $N_{0}\left(t_{\mathrm{d}}+t_{\mathrm{f}}\right) / N_{0 \text {, sat }}=0.8$, respectively [27]. As shown in Fig. 3(b), $t_{\mathrm{d}}$ follows a nice linear increasing function of $t_{\text {ramp }}$ for all the quenches. However, as $t_{\text {ramp }}$ increases, $t_{\mathrm{f}}$ first decreases until it reaches a minimum around $t_{\text {ramp }} \approx 400 \mathrm{~ms}$, and then increases linearly. Based on this observation, we classify the quenches into two types, normal and fast ones, which are separated at $t_{\text {ramp }} \sim 500 \mathrm{~ms}$ for our system. By plotting $N_{0}(t) / N_{0 \text {, sat }}$ vs $\left(t-t_{\mathrm{d}}\right) / t_{\mathrm{f}}$, we find that all experimental data for normal quenches can be well described by a single universal curve [see Fig. 3(c)], while those for fast quenches exhibit a significant deviation from this curve [Fig. 3(d)].

\section{KZ SCALING OF SPONTANEOUS VORTICES}

We now study the spontaneous generation of vortices in the quench dynamics, by measuring the vortex density $\rho_{v}$ at the saturation of the quasicondensate for each $t_{\text {ramp. It is }}$ known that near the superfluid transition $T_{\mathrm{c}}$, a diverging correlation length develops as $\xi \sim\left|T-T_{\mathrm{c}}\right|^{-v}$ and the relaxation time diverges as $\tau \sim \xi^{z}$, with $v$ and $z$ being the static and dynamic critical exponents, respectively $[18,19]$. Under the condition that the temperature $T$ varies linearly with time near $T_{\mathrm{c}}$, the KZ theory predicts that $\rho_{v}$ decays algebraically with the quench rate $1 / \tau_{\mathrm{Q}}$ as $\rho_{v} \sim \tau_{\mathrm{Q}}^{-\alpha_{\mathrm{KZ}}}$, where the exponent $\alpha_{\mathrm{KZ}}$ is determined by $v$ and $z$. Experimentally, the measurement of temperature evolution in the quench dynamics is a great challenge for strongly interacting Fermi gases. In previous

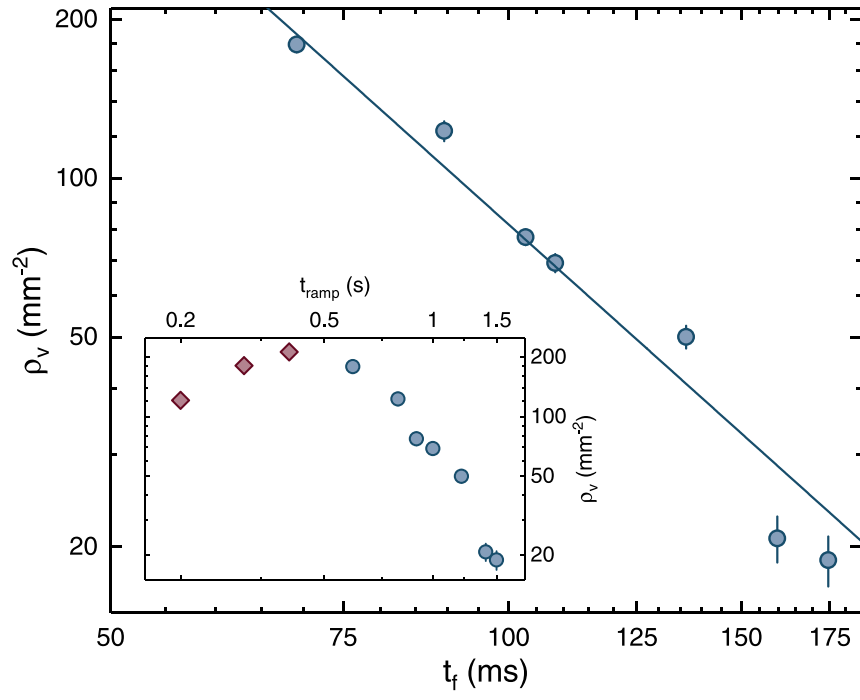

FIG. 4. Log-log plot of vortex density $\rho_{v}$ vs $t_{\mathrm{f}}$. The error bar for each point represents the standard statistical error over 30 independent measurements. The solid straight line is the power-law fitting curve based on the KZ theory. The inset shows $\rho_{v}$ as a function of $t_{\text {ramp }}$, where blue circles and red squares denote normal and fast quenches, respectively.

studies, $\tau_{\mathrm{Q}} \sim t_{\text {ramp }}$ has been reported [13,20,28,29], and thus

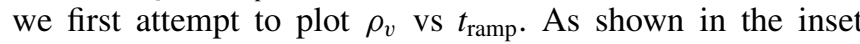
of Fig. 4, an approximate power-law decay is observed for normal quenches, while for fast quenches the $t_{\text {ramp }}$ dependence of $\rho_{v}$ clearly deviates from the KZ scaling.

To understand the normal and fast quenches better, we revisit the relaxation dynamics of an out-of-equilibrium system. For a superfluid, there are two types of excitations, i.e., low-energy density waves and high-energy vortices. Typically, the relaxation of low-energy modes is much faster than the annihilation of vortex and antivortex pairs. The quasiequilibrium condition is assumed that, at each evolution time, the low-energy modes have been sufficiently relaxed while the vortices remain excited. For normal quenches, the quasiequilibrium condition is supported by the observations that the formation time $t_{\mathrm{f}}$ of the quasicondensate increases linearly with $t_{\text {ramp }}$ [see Fig. 3(b)], $N_{0}$ almost reaches $N_{0 \text {, sat }}$ at the end of the quench, and that the saturated vortex density $\rho_{v}$ decays algebraically. On the other hand, the unusual $t_{\text {ramp }}$ dependence of $t_{\mathrm{f}}$ and $\rho_{v}$, as well as the barely started growth of $N_{0}$ by $t=t_{\text {ramp }}$, suggest that the quasiequilibrium condition is broken for fast quenches.

In Fig. 4 , the data points of $\rho_{v}$ vs $t_{\mathrm{f}}$ for normal quenches agree well with a power-law scaling. Indeed, $t_{\mathrm{f}}$ reflects the linear growth period of $N_{0}$ and the linear decrease of temperature with time. Unlike $t_{\mathrm{ramp}}$, it is insensitive to the (somewhat arbitrary) initial temperature of the system ( $T$ at $t=0)$ as well as the complications caused by the pair-formation process during the slow incubation stage. Therefore, it is inversely proportional to the quench rate near $T_{\mathrm{c}}$ and thus may naturally play the role of $\tau_{\mathrm{Q}}$ in the $\mathrm{KZ}$ theory. Fitting the experimental data with a power-law function $\rho_{v} \sim t_{\mathrm{f}}^{-\alpha_{\mathrm{KZ}}}$, we obtain the $\mathrm{KZ}$ exponent $\alpha_{\mathrm{KZ}}=2.25(17)$. In a 3D harmonic trap, the $\mathrm{KZ}$ exponent has been predicted to be $\alpha_{\mathrm{KZ}}=2(1+2 v) /(1+$ 
vz) [30,31]. According to the $F$ model, $v=2 / 3$ and $z=3 / 2$ for a 3D system [32], which yields $\alpha_{\mathrm{KZ}}=7 / 3$. Our experimental result is in quantitative agreement with this theoretical value, demonstrating the validity of using $t_{\mathrm{f}}$ to characterize the quench rate for normal quenches.

\section{CONCLUSIONS}

In conclusion, we have studied the quench dynamics of a strongly interacting atomic Fermi gas by ramping down the trapping potential. Our experiment directly demonstrates the interplay between the real-time dynamics of quasicondensate growth and spontaneous vortex formation. The good agreement between theoretical calculations and experimental data demonstrates that our data can be explained by the pseudogap physics, which leads to significant differences in the growth dynamics of the quasicondensate between the BEC and BCS regimes. We find that the quench processes can be classified into normal and fast quenches. The unusual nonmonotonic $t_{\text {ramp }}$ dependence of the quasicondensate formation time $t_{\mathrm{f}}$ and the vortex density $\rho_{v}$ suggests that the quasiequilibrium condition is broken during the fast quench processes. For normal quenches, by using $t_{\mathrm{f}}$ to characterize the quench time of the system, the KZ scaling of strongly interacting Fermi gas is observed and the extracted KZ exponent agrees well with the theoretical prediction. Our work may serve as a starting point for exploring rich quantum phenomena of quasi-two-dimensional (2D) vortices, such as Berezinskii-Kosterlitz-Thouless physics in a quasi-2D trap [33], holographic liquids [34], and quantum turbulence [35-37].

\section{ACKNOWLEDGMENTS}

This work is supported by the National Key R\&D Program of China (Grants No. 2018YFA0306501, No. 2017YFA0304204, and No. 2016YFA0301604), NSFC of China (Grants No. 11874340 , No. 11425417, No. 11774067 , No. 11774309, No. 11625522, and No. 11934002), the Chinese Academy of Sciences (CAS), the Anhui Initiative in Quantum Information Technologies, and the Shanghai Municipal Science and Technology Major Project (Grant No. 2019SHZDZX01).
[1] I. Bloch, J. Dalibard, and W. Zwerger, Many-body physics with ultracold gases, Rev. Mod. Phys. 80, 885 (2008).

[2] S. Giorgini, L. P. Pitaevskii, and S. Stringari, Theory of ultracold atomic Fermi gases, Rev. Mod. Phys. 80, 1215 (2008).

[3] C. Chin, R. Grimm, P. Julienne, and E. Tiesinga, Feshbach resonances in ultracold gases, Rev. Mod. Phys. 82, 1225 (2010).

[4] T. Esslinger, Fermi-Hubbard physics with atoms in an optical lattice, Annu. Rev. Condens. Matter Phys. 1, 129 (2010).

[5] Q. Chen and J. Wang, Pseudogap phenomena in ultracold atomic Fermi gases, Front. Phys. 9, 539 (2014).

[6] E. J. Mueller, Review of pseudogaps in strongly interacting Fermi gases, Rep. Prog. Phys. 80, 104401 (2017).

[7] T. Timusk and B. Statt, The pseudogap in high-temperature superconductors: An experimental survey, Rep. Prog. Phys. 62, 61 (1999).

[8] P. A. Lee, N. Nagaosa, and X.-G. Wen, Doping a Mott insulator: Physics of high-temperature superconductivity, Rev. Mod. Phys. 78, 17 (2006).

[9] P. A. Lee, From high temperature superconductivity to quantum spin liquid: Progress in strong correlation physics, Rep. Prog. Phys. 71, 012501 (2007).

[10] G. Baym, C. Pethick, and D. Pines, Superfluidity in neutron stars, Nature (London) 224, 673 (1969).

[11] L. E. Sadler, J. M. Higbie, S. R. Leslie, M. Vengalattore, and D. M. Stamper-Kurn, Spontaneous symmetry breaking in a quenched ferromagnetic spinor Bose-Einstein condensate, Nature (London) 443, 312 (2006).

[12] C. N. Weiler, T. W. Neely, D. R. Scherer, A. S. Bradley, M. J. Davis, and B. P. Anderson, Spontaneous vortices in the formation of Bose-Einstein condensates, Nature (London) 455, 948 (2008).

[13] G. Lamporesi, S. Donadello, S. Serafini, F. Dalfovo, and G. Ferrari, Spontaneous creation of Kibble-Zurek solitons in a Bose-Einstein condensate, Nat. Phys. 9, 656 (2013).
[14] L. Corman, L. Chomaz, T. Bienaimé, R. Desbuquois, C. Weitenberg, S. Nascimbène, J. Dalibard, and J. Beugnon, Quench-Induced Supercurrents in an Annular Bose Gas, Phys. Rev. Lett. 113, 135302 (2014).

[15] N. Navon, A. L. Gaunt, R. P. Smith, and Z. Hadzibabic, Critical dynamics of spontaneous symmetry breaking in a homogeneous Bose gas, Science 347, 167 (2015).

[16] L. Chomaz, L. Corman, T. Bienaimé, R. Desbuquois, C. Weitenberg, S. Nascimbène, J. Beugnon, and J. Dalibard, Emergence of coherence via transverse condensation in a uniform quasi-two-dimensional Bose gas, Nat. Commun. 6, 6162 (2015).

[17] M. Anquez, B. A. Robbins, H. M. Bharath, M. Boguslawski, T. M. Hoang, and M. S. Chapman, Quantum Kibble-Zurek Mechanism in a Spin-1 Bose-Einstein Condensate, Phys. Rev. Lett. 116, 155301 (2016).

[18] T. W. B. Kibble, Topology of cosmic domains and strings, J. Phys. A: Math. Gen. 9, 1387 (1976).

[19] W. H. Zurek, Cosmological experiments in superfluid helium? Nature (London) 317, 505 (1985).

[20] B. Ko, J. W. Park, and Y. Shin, Kibble-Zurek universality in a strongly interacting Fermi superfluid, Nat. Phys. 15, 1227 (2019).

[21] Q. J. Chen, I. Kosztin, B. Jankó, and K. Levin, Pairing Fluctuation Theory of Superconducting Properties in Underdoped to Overdoped Cuprates, Phys. Rev. Lett. 81, 4708 (1998).

[22] Q. J. Chen, J. Stajic, S. N. Tan, and K. Levin, BCS-BEC crossover: From high temperature superconductors to ultracold superfluids, Phys. Rep. 412, 1 (2005).

[23] X.-C. Yao, H.-Z. Chen, Y.-P. Wu, X.-P. Liu, X.-Q. Wang, X. Jiang, Y. Deng, Y.-A. Chen, and J.-W. Pan, Observation of Coupled Vortex Lattices in a Mass-Imbalance Bose and Fermi Superfluid Mixture, Phys. Rev. Lett. 117, 145301 (2016). 
[24] The quasicondensate formation rate scales roughly as $1 / T_{\mathrm{c}}$, which increases monotonically from the BEC to the BCS regimes in a harmonic trap.

[25] Q. Chen, J. Stajic, and K. Levin, Thermodynamics of Interacting Fermions in Atomic Traps, Phys. Rev. Lett. 95, 260405 (2005).

[26] For a qualitative comparison between experiment and theory, fine tuning of $\Omega_{\mathrm{c}}$ is not necessary.

[27] Note that a slight variation in the cutoff percentages does not change the power-law exponent in the KZ scaling in Fig. 4.

[28] S. Donadello, S. Serafini, T. Bienaimé, F. Dalfovo, G. Lamporesi, and G. Ferrari, Creation and counting of defects in a temperature-quenched Bose-Einstein condensate, Phys. Rev. A 94, 023628 (2016).

[29] J. Goo, Y. Lim, and Y. Shin,, Defect Saturation in a Rapidly Quenched Bose Gas, Phys. Rev. Lett. 127, 115701 (2021).

[30] W. H. Zurek, Causality in Condensates: Gray Solitons as Relics of BEC Formation, Phys. Rev. Lett. 102, 105702 (2009).

[31] A. del Campo, A. Retzker, and M. B. Plenio, The inhomogeneous Kibble-Zurek mechanism: Vortex nucleation during Bose-Einstein condensation, New J. Phys. 13, 083022 (2011).
[32] P. C. Hohenberg and B. I. Halperin, Theory of dynamic critical phenomena, Rev. Mod. Phys. 49, 435 (1977).

[33] V. L. Berezinskii, Destruction of long-range order in one dimensional and two dimensional systems possessing a continuous symmetry group II. Quantum systems, Zh. Eksp. Teor. Fiz. 61, 1144 (1971) [Sov. Phys. JETP 34, 610 (1972)]; J. M. Kosterlitz and D. J. Thouless, Ordering, metastability and phase transitions in two dimensional systems, J. Phys. C: Solid State Phys. 6, 1181 (1973).

[34] P. M. Chesler, H. Liu, and A. Adams, Holographic vortex liquids and superfluid turbulence, Science 341, 368 (2013).

[35] M. Tsubota, Quantum turbulence: From superfluid helium to atomic Bose-Einstein condensates, Contemp. Phys. 50, 463 (2009).

[36] C. F. Barenghi, L. Skrbek, and K. R. Sreenivasan, Introduction to quantum turbulence, Proc. Natl. Acad. Sci. USA 111, 4647 (2014).

[37] A. Bulgac, M. M. Forbes, and G. Wlazłowski, Towards quantum turbulence in cold atomic fermionic superfluids, J. Phys. B: At., Mol. Opt. Phys. 50, 014001 (2016). 